Ann. Biol. anim. Bioch. Biophys., 1966, 6 (I), 33-46.

\title{
METABOLISME DE LA FLORE INTESTINALE DU PORC DÉGRADATION DES FORMES L ET D DES ACIDES AMINÉS
}

\author{
M. C. MICHEL \\ avec la collaboration technique de Simone Boche \\ Laboratoire des Métabolismes \\ Centre national de Recherches zootechniques, 78 - Jouy-en-Josas
}

SOMMAIRE

In vitro, la flore microbienne intestinale clu porc catabolise tous les acides aminés. L'arginine les acides glutamique et aspartique, la sérine et la thréonine sont dégradés le plus rapidement. La proline et le tryptophane sont faiblement attarqués.

Cette réaction est spécifique de la forme $I$, pour les acides aminés indispensables dont la forme D n'est pas dégradée (sauf la D-méthionine). La forme I) des acides aminés semi-indispensables et indifférenciés est catabolisée moins rapidement que l'isomère $L$.

I.es substances azotées qui résultent de cette dégradation sont de l'ammoniac dans tous les cas, ainsi que des acides aminés (ornithine et citrulline à partir de l'arginine) et des amines (cadavérine, putrescine, tyramine, tryptamine et histamine).

L'activité de la flore est maximum dans l'iléon et le cæcum. $A$ un même niveau intestinal, l'activité est soumise à des variations saisonnières (maximum en été) qui se reproduisent d'une année à l'autre.

L'influence éventuelle de cette action microbienne dans l'animal vivant est discutée.

La flore microbienne intestinale du porc dégrade in vitro beaucoup de substances azotées, et en particulier tous les acides aminés, par désamination et décarboxylation (MICHEL, Ig6I a). L'existence de ce catabolisme a été démontrée chez l'animal vivant par LARson et HILL ( 1960 ) ; ces auteurs ont identifié diverses amines, produit de la décarboxylation microbienne des acides aminés dans l'iléon du jeune porc. La formation de ces substances est fonction de l'âge, de la nutrition et de l'état physiologique de l'animal. Michel et al. (I964), ont observé que l'excrétion fécale des bases azotées (cadavérine, putrescine et ammoniac en majorité), pratiquement

Annales de Biologie animale. - 1966. 
nulle chez le porcelet allaité, s'accroissait très rapidement après le sevrage et atteignait une valeur élevée pendant les diarrhées. D'autres variations de l'activité catabolique de la flore intestinale ont été mises en évidence. Par exemple, celle-ci (mesurée in vitro), atteint un maximum pendant l'été. C'est pendant cette période que le taux d'ammoniac du sang de la veine porte est également le plus élevé (FraNÇOIS et MICHEI, Ig60).

Meinicowyckz et Johansson (I955), ont mis en évidence in vivo et in vitro la formation d'amines par la flore intestinale du rat. L'activité de cette dernière s'exerce également à l'égard d'autres substances azotées : le contenu cæcal du rat aseptique comprend une quantité importante d'urée et de glutamine, qui sont absentes dans le cæcum de l'animal conventionnel; en revanche, chez ce dernier on observe la présence de diverses bases azotées (MICHEL et SACQUET, I965).

Chez le ruminant, EL-ShazLy (I952), a montré in vivo que la flore du rumen du mouton dégradait les protéines avec formation d'acides volatils et de $\mathrm{NH}_{3}$. Ces résultats ont été observés in vitro par LEWIS (I955), chez le mouton, et LEwIS et EMERY (Ig62), chez la vache.

Afin de faire la part de ces diverses actions dans la digestion de 1'hôte, le but de cette étude est d'étendre et préciser nos travaux antérieurs, en ce qui concerne l'activité catabolique de la flore intestinale du porc à l'égard des acides aminés. Il s'agit de déterminer l'activité potentielle de la flore, ses variations, et de préciser les différents schémas de dégradation possible.

\section{MÉTHODES}

Isolement de la flore intestinale.

Dès l'abatage de l'animal (porcs de go à roo kg), le contenu du cæcum est prélevé et homogénéisé. On pèse $100 \mathrm{~g}$ de cette suspension et on mélange les prélèvements effectués sur to animaux. Le prélèvement moyen est dilué au I/2 (poids/volume) par de l'eau distillée, puis après homogénéisation à l'agitateur, filtré sur une toile de nylon très fine. Ceci permet d'éliminer la majeure partie des particules alimentaires. Le filtrat est conservé à $+4^{\circ} \mathrm{C}$ jusqu'au moment de l'emploi, 4 jours au maximum; (une température inférieure à $0^{\circ} \mathrm{C}$ inactive les actions enzymatiques).

\section{Incubation.}

La suspension est diluée $(V / V)$, dans le substrat d'acide aminé $M / 25$. On fait incuber à l'étuve à $37^{\circ} \mathrm{C}$ en tubes fermés.

\section{Analyses.}

La suspension microbienne contient une faible quantité d'acides aminés, peptides, bases aminées, et $\mathrm{NH}_{3}$. Ces valeurs sont déterminées par l'analyse d'un témoin sans acide aminé et déduites des résultats des échantillons contenant des acides aminés ajoutés. L'analyse est effectuée avant incubation puis après $16 \mathrm{~h}$ de la manière suivante : on porte $20 \mathrm{ml}$ de l'échantillon au bain-marie à $100^{\circ} \mathrm{C}$ pendant $5 \mathrm{mn}$, puis après refroidissement, on centrifuge $25 \mathrm{mn}$ à $9000 \mathrm{~g}$. Le surnageant limpide est stocké à $-{ }_{1} 5^{\circ} \mathrm{C}$ jusqu'au moment du dosage, d'après les techniques suivantes :

I $^{\circ}$ Dosage de l'azote alpha-aminé (MICHEL, I96r $c$ ).

$2^{\circ}$ Dosage de l'azote ammoniacal (CoNWAY, I957).

$3^{0}$ Dosage des acides aminés et des amines par chromatographie sur résine échangeuse de cations (MICHEL, à paraître). 


\section{RÉSULTATS}

\section{I $^{\circ}$ Étude des conditions d'incubation}

Afin de déterminer in vitro l'activité catabolique potentielle de la flore microbienne à l'égard de divers substrats, il convient de se placer dans des conditions physico-chimiques proches, sinon identiques, de celles qui existent au niveau intestinal. En effet, la synthèse d'enzymes adaptatifs au cours de la croissance microbienne, telles que les décarboxylases, est conditionnée par le $\mathrm{pH}$ du milieu de culture (GALE, I946). De plus, les proportions respectives d'enzyme et de substrat, la valeur du potentiel d'oxydo-réduction, déterminent le sens des réactions (WURMSER, I935).

Les valeurs du pH et du potentiel d'oxydo-réduction, mesurées sur des contenus intestinaux prélevés sur des animaux à l'abatage sont rapportées dans le tableau I.

TABLEAU I

\begin{tabular}{|c|c|c|}
\hline & pH & Eh \\
\hline Intestin grêle & $6,10 \pm 0,3$ & $-225 \mathrm{mV}+30$ \\
\hline Cæcum & $5,79 \pm 0,45$ & $-225 \mathrm{mV} \pm 45$ \\
\hline
\end{tabular}

(Valeurs moyennes sur 20 animaux).

\section{TABLEAU 2}

Teneurs du contenu intestinal du porc en matières azotées, glucose, germes microbiens et activité de ceux-ci

(Valeurs moyennes sur 40 porcs)

\begin{tabular}{|c|c|c|c|c|c|}
\hline \multirow{2}{*}{$\begin{array}{l}\text { Localisation } \\
\text { anatomique }\end{array}$} & \multicolumn{4}{|c|}{ Concentration en $\mathrm{g} / \mathrm{kg}$ frais } & \multirow{2}{*}{ Activité (1) } \\
\hline & Glucose & $\mathrm{N}-\alpha$ aminé libre & $\mathrm{N}\left(\mathrm{NII}_{3}\right)$ & Microbes $(\mathrm{g} / \mathrm{sec})$ & \\
\hline Estomac $\ldots . . \ldots$ & 1,59 & 0,45 & 0,19 & 1,5 & 0,45 \\
\hline Duodénum-Jéjunum & 3,88 & 1,27 & 0,13 & 1,5 & 0,63 \\
\hline Iléon $\ldots \ldots \ldots \ldots$ & 2,64 & 0,68 & 0,15 & 3 & 0,84 \\
\hline Cæcum ......... & $<0,1$ & 0,05 & 0,30 & 8 & 0,95 \\
\hline Colon $\ldots \ldots \ldots \ldots$ & $<0,1$ & 0,08 & 0,50 & 13,5 & 0,93 \\
\hline
\end{tabular}

(1) Exprimée en millimoles de $\mathrm{N}\left(\mathrm{NH}_{3}\right)$ libérée par heure et par g de poids sec microbien à partir d'une solution d'arginine $\mathrm{M} / 50$. 
Les valeurs du $\mathrm{pH}$ sont en bon accord avec celles trouvées par MOORE et TYLER (1955) et L,UDWIGSEN et THORBECK (I96r); ces derniers auteurs avaient effectué les prélèvements à 1'aide d'une canule posée sur le cæcum.

Des microbes sont présents dans les différentes parties du tractus gastro-intestinal ; leur quantité croît de l'estomac au rectum. Ce n'est qu'à partir de l'iléon que leur activité biochimique (mesurée in vitro par la vitesse de dégradation de l'arginine) est maximum. C'est également à partir de l'iléon que le taux de glucides libres tend vers zéro. La teneur intestinale en ammoniac s'accroît en même temps que l'activité catabolique des germes (tabl. 2).

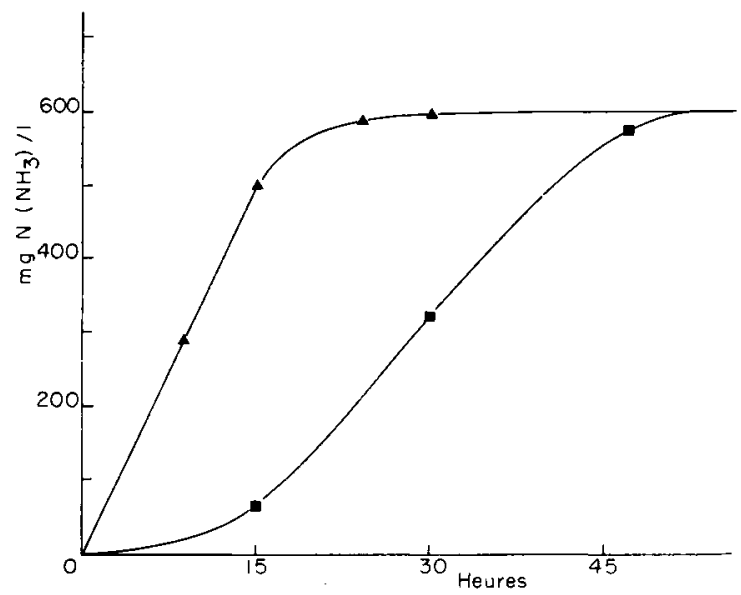

Fig. 1. - Influence du mode de préparalion de la suspension microbienne sur l'activité enzymatique

- a) Flore du cæcum diluée au $\mathrm{I} / 4$.

- b) Flore à la même concentration centrifugée et lavée deux fois.

Arginine $\mathrm{M} / 5 \mathrm{O}$.

En ce qui concerne le mode de préparation des suspensions microbiennes, celui-ci influe sur 1'activité enzymatique. Par exemple, des techniques bactériologiques classiques (centrifugation, lavage des germes) diminuent cette activité. La figure I indique la cinétique de dégradation de l'arginine par la flore, diluée directement dans le substrat $(a)$, ou centrifugée et lavée $(b)$. La courbe en $\mathrm{S}$, obtenue avec le procédé $(b)$, ne permet pas d'apprécier la vitesse de dégradation ; alors qu'avec la méthode $(a)$, on observe une dégradation de vitesse constante qui débute dès la mise en incubation. Dans les deux cas, cependant, le résultat final est identique.

$\mathrm{Si}$ on considère l'influence respective de la concentration des microbes et du substrat, on observe que lorsque cette dernière est constante $(0,04 \mathrm{M} / 1$ itre $)$, la vitesse de dégradation est une fonction linéaire de la teneur en microbes, (fig. 2). Elle est également linéaire en fonction de la concentration du substrat, mais jusqu'à o,or M/litre. Au-dessus de o,o2 M/litre. La quantité de $\mathrm{N}\left(\mathrm{NH}_{3}\right)$ libérée est constante quelle que soit la concentration du substrat, (fig. 3). De ce fait, ni le substrat, ni les produits de la réaction ne sont inhibiteurs.

En phase proliférante, l'activité catabolique globale est faible ; la diminution du taux des acides aminés du milieu est inversement proportionnelle à la formation 


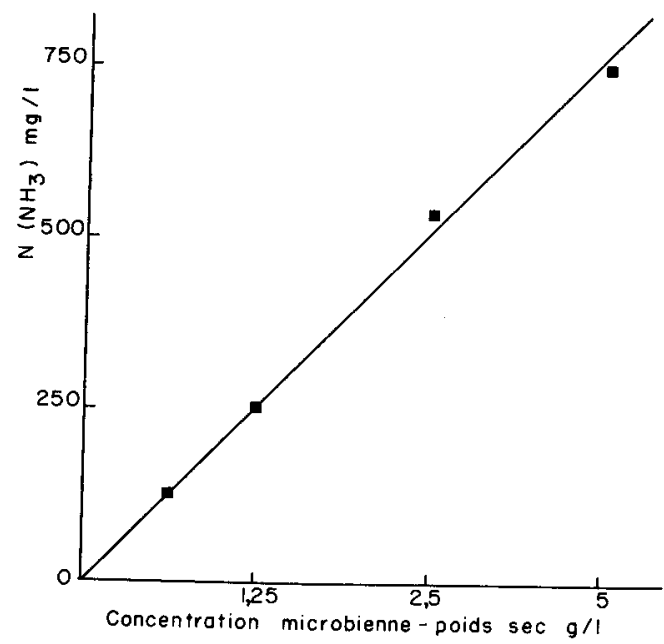

FIG. 2. - Influence de la densité microbienne sur la vitesse de dégradation de l'arginine (mesurée par l'ammoniac libéré)

Arginine $\mathrm{M} / 25$.

Durée d'incubation I6 heures.

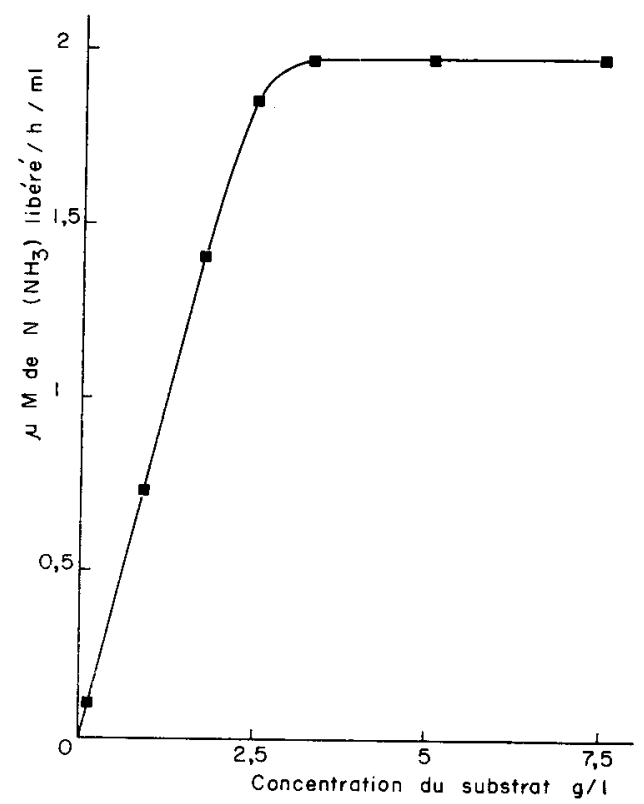

Iig. 3. - Relation entre la concenlration du substrat et la libération d'ammoniac Flore du cæecum diluée au $\mathrm{r} / 4$. Durée d'incubation 16 heures. 
de corps microbiens. Le tatx de $\mathrm{NH}_{3}$ diminue vraisemblablement par amination du pyruvate formé au cours de la glycolyse. Ceci n'exclut pas que certains acides aminés telle que l'arginine, puissent être dégradés (KNiveiTt, I960). A la fin de la croissance, l'autolyse des corps microbiens s'accompagne d'une importante formation d'ammoniac (fig. 4).

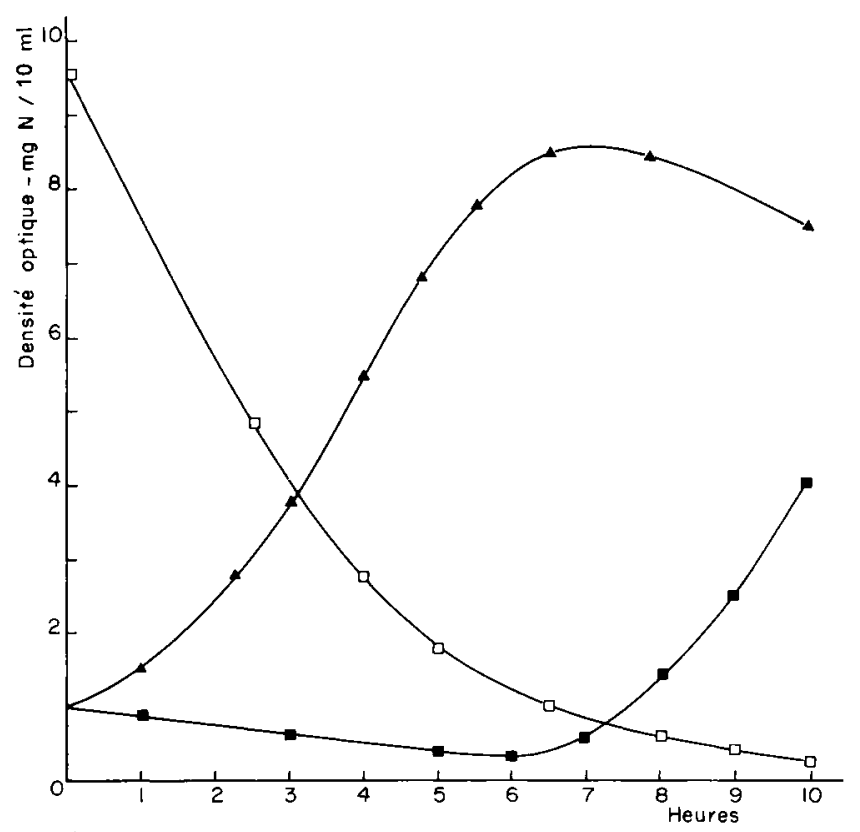

FIG. 4. - Évolution de la teneur en azote $\alpha$ aminé et en azote ammoniacal au cours des phases du métabolisme microbien

- densité optique de la suspension.

$\square$ azote $\alpha$ aminé ( $\mathrm{mg} / \mathrm{lo} \mathrm{ml}$ )

- - azote ammoniacal ( $\mathrm{mg} / \mathrm{ro} \mathrm{ml}$ ).

\section{$2^{\circ}$ Catabolisme des divers acides aminés}

a) Dégradation par désamination.

La vitesse de dégradation des différents acides aminés, mesurée à la fois par le pourcentage d'ammoniac libéré et celui de l'acide aminé disparu, varie dans de larges limites. On ne peut établir de relation entre la structure chimique du substrat et la vitesse de dégradation : celle-ci est maximum pour des substances aussi dissemblables que 1'arginine, 1'acide glutamique et la sérine.

On observe dans tous les cas une libération d'ammoniac, mais d'autres substances peuvent être produites (acides aminés et amines). Ces résultats sont reportés dans le tableau 3 .

b) Dégradation par décarboxylation.

Le catabolisme anaérobie des acides aminés produit outre $\mathrm{NH}_{3}$, une certaine quantité de $\mathrm{CO}_{2}$. Ce dernier peut provenir de la coupure du carboxyle du groupe- 
ment $\alpha$ aminé. Diverses espèces microbiennes, Escherichia coli, Streptococus faecalis, Clostridia, Proteus, etc.) décarboxylent six acides aminés de forme $\mathrm{L}$ (lysine, ornithine, arginine, tyrosine, histidine et acide glutamique) à l'exclusion des autres (GALE I946). Il en résulte la production de l'amine correspondante et du $\mathrm{CO}_{2}$. A part l'acide $\gamma$-amino-butyrique que nous n'avons pu mettre en évidence en

TABI,EAU 3

Dégradation des acides aminés par la flore du icecum du pore

(Substrat M/50-flore au $1 / 4-\mathrm{I} 6 \mathrm{~h}$ d'incubation)

Essais sur 80 animaux abattus en hiver

\begin{tabular}{|c|c|c|c|}
\hline Acides aminés & $\begin{array}{l}\text { P. } 100 \mathrm{~N}\left(\mathrm{NH}_{3}\right) \text { alparu } \\
\text { calculé sur le } \\
\text { groupement } \alpha \text { anniné }\end{array}$ & $\begin{array}{l}\text { P. } 100 \text { acide :muiné } \\
\text { disjraru }\end{array}$ & $\begin{array}{c}\text { Substances azotées } \\
\text { produites } \\
\text { autres que } \mathrm{NH}_{3}\end{array}$ \\
\hline Arginine $\ldots \ldots \ldots \ldots \ldots \ldots \ldots$ & 188 & 72 & $\begin{array}{l}\text { Agnatine, ornithine, } \\
\text { citrulline, putrescine. }\end{array}$ \\
\hline Acide glutamique........... & 100 & 100 & \\
\hline Sérine $\ldots \ldots \ldots \ldots \ldots \ldots \ldots$ & 100 & 100 & \\
\hline Acide aspartique . . . . . . . . & $7 !$ & 78 & \\
\hline Thréonine $\ldots \ldots \ldots \ldots \ldots \ldots$ & 69,8 & 62 & \\
\hline 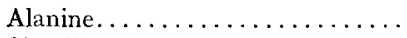 & 47,2 & 50 & \\
\hline Citrulline $\ldots \ldots \ldots \ldots \ldots \ldots \ldots$ & 45,8 & $: 38$ & Ornithine, putrescine. \\
\hline Leucine $\ldots \ldots \ldots \ldots \ldots \ldots \ldots$ & 41 & 52 & \\
\hline Isoleucine .................. & 33 & 42 & \\
\hline Histidine $\ldots \ldots \ldots \ldots \ldots \ldots$ & 32,8 & 98 & $\begin{array}{l}\text { Acide urocanique, his- } \\
\text { tamine. }\end{array}$ \\
\hline Cystine $\ldots \ldots \ldots \ldots \ldots \ldots \ldots$ & 31 & & \\
\hline Glycocolle $\ldots \ldots \ldots \ldots \ldots \ldots$ & 28,4 & $\underline{98}$ & \\
\hline Valine..$\ldots \ldots \ldots \ldots \ldots \ldots$ & 25,4 & 3 & \\
\hline Ornithine $\ldots \ldots \ldots \ldots \ldots \ldots$ & 15 & 28 & Putrescine. \\
\hline 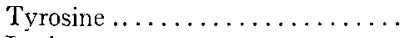 & 13,6 & 22 & 'Iyramine. \\
\hline Lysine $\ldots \ldots \ldots \ldots \ldots \ldots \ldots$ & 12 & $\because 1$ & Cadavérine. \\
\hline Phénylalanine ............. & 11 & 30 & \\
\hline Tryptophane ................ & 10 & 15 & Indole, tryptamine. \\
\hline Néthionine.............. & 10 & 20 & \\
\hline Proline .................. & 6 & & \\
\hline
\end{tabular}

tant que produit terminal de la dégradation de l'acide glutamique, la flore intestinale du porc produit ces diverses amines, ainsi que l'indique le tableau 3. A partir des autres acides aminés, on observe une certaine formation de $\mathrm{CO}_{2}$, mais sans que l'amine correspondante soit le produit terminal. Il est probable, d'après la cinétique de la dégradation, que l'acide aminé est d'abord désaminé puis le résidu carboné est dégradé à son tour. Les acides aminés qui ne produisent pas d'amines se comportent à cet égard comme la L-thréonine, (fig. 5).

c) Spécificité stéréochimique de ces réactions de dégradation.

Les acides aminés indispensables de forme $I_{1}$ sont seuls décarboxylés par 1a flore. Parmi les isomères $\mathrm{D}$, seule la $\mathrm{D}$-méthionine est dégradée. La réaction de dé- 
carboxylation ne débute qu'après un temps de latence prolongé ( 7 heures). On observe également une libération du $\mathrm{NH}_{3}$ à partir du dérivé $\mathrm{D}$, mais à vitesse beaucoup plus faible que pour la forme $\mathrm{L}$.

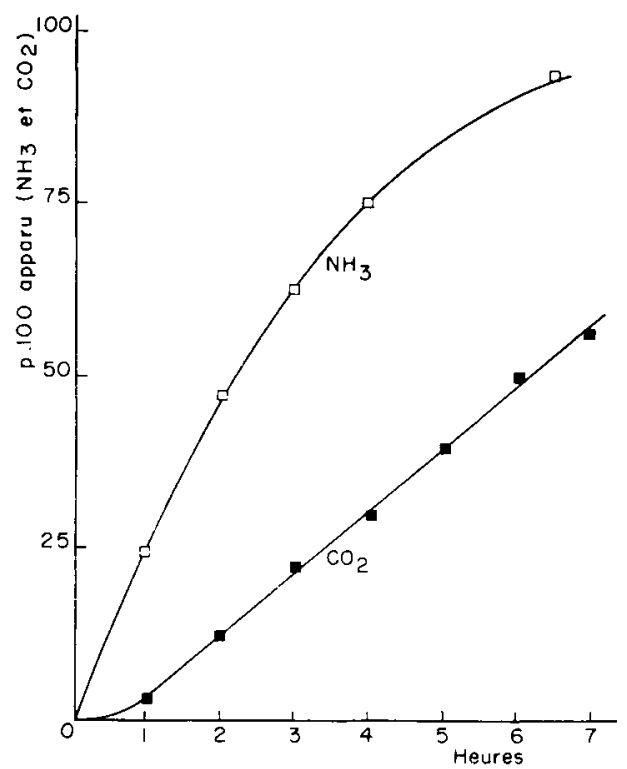

FIG. 5. - Dégradation de la L-thréonine

Solution $\mathrm{M} / \mathrm{roo}$. Flore au $\mathrm{I} / 4$. Incubation dans l'appareil de Warburg.

$\mathrm{CO}^{2}$ : pourcentage obtenu par rapport à la théorie (moyenne de 3 fioles) ; le $\mathrm{CO}^{2}$ formé par la suspension sans acide aminé a été déduit.

$\hat{N}\left(\mathrm{NH}_{3}\right)$ : valeur moyenne de deux essais successifs. Après le temps d'incubation considéré, deux fioles sont retirées et l'ammoniac dosé par Conway.

TABLEAU 4

Dégradation des formes $L$ et $D$ des acides aminés essentiels

\begin{tabular}{|c|c|c|c|c|}
\hline \multirow[b]{2}{*}{ Acides anninés } & \multicolumn{2}{|c|}{ Isomére $\mathrm{L}$ * } & \multicolumn{2}{|c|}{ Isomère $\mathrm{D} *$} \\
\hline & $\mathrm{NH}_{3}$ & $\mathrm{CO}_{2}$ & $\mathrm{NH}_{3}$ & $\mathrm{CO}_{2}$ \\
\hline Histidine $\ldots \ldots \ldots \ldots \ldots$ & 112 & $10:$ & 78,5 & 51 \\
\hline Thréonine...$\ldots \ldots \ldots \ldots$ & 95 & 55 & 0 & 6 \\
\hline Leucine .............. & 81 & 76 & 0 & t \\
\hline Isoleucine ... . . . . . . . . & 78 & j6 & 0 & 0 \\
\hline Lysine ............... & 48 & $\therefore 5$ & 5 & 9 \\
\hline Valine ............... & 40 & 57 & 0 & 0 \\
\hline Méthionine .......... & 30 & $102 * *$ & 10 & $49^{* *}$ \\
\hline Tryptophane . . . . . . . . . & 17 & 38 & 0 & 0 \\
\hline Phénylalanine ......... & 9 & 5 & 0 & 0 \\
\hline Proline ............... & 0 & 5 & & \\
\hline
\end{tabular}

* Pourcentage de dégradation calculé par rapport au groupement alpha-aminé. Solution M/50. Incubation 16 h à $37^{\circ} \mathrm{C}$. Flore diluée au $1 / t^{\prime}$.

** $\mathrm{Y}$ compris $\mathrm{H}_{2} \mathrm{~S}$. 
En ce qui concerne les acides aminés semi-indispensables et indifférenciés (histidine, alanine, sérine, acides glutamique et aspartique) la forme $D$ est dégradée

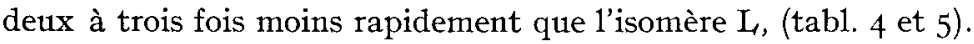

TABLEAU 5

Dégradation de quelques acides aminés non indispensables

(Mesure de $\mathrm{NII}_{3}$ )

\begin{tabular}{|c|c|c|c|}
\hline Alanine $\mathrm{J} . .$. & 75 & Acide aspartique $\mathrm{L}$. & 98 \\
\hline Alanine $\mathrm{D} \ldots \ldots$. & 25 & Acide aspartique $D$. & 97 \\
\hline Alanine $\mathrm{DL} \ldots \ldots$ & $5 \cdot 1$ & Acide glutamique $\mathrm{L}$. & 100 \\
\hline Sérine DL. . . . . . & 85 & Acide glutamique D. & 90 \\
\hline
\end{tabular}

Conditions identiques à celles du tableau ' 1 .

d) Racémisation.

I a lysine peut être racémisée par des espèces microbiennes (Proteus et Escherichia), d'après la réaction suivante : L-1ysine $\rightleftarrows$ D-1ysine (HuANG et Davissor, r958). Dans nos conditions opératoires, la flore intestinale du porc ne possède pas cette propriété (tabl. 6), bien que le genre Escherichia soit largement présent au niveau intestinal (Dickinson et al. I96I).

Dans d'autres conditions ( $\mathrm{pH} 8,6$ ) durée d'incubation $40 \mathrm{~h}$, on observe une légère action ( 20 p. IOO) mais ces conditions ne sont pas physiologiques. Il faut noter que pour les acides aminés essentiels de forme $D$, l'inertie du groupement alphaaminé à la dégradation microbienne permet de supposer qu'il n'existe pas de racémisation.

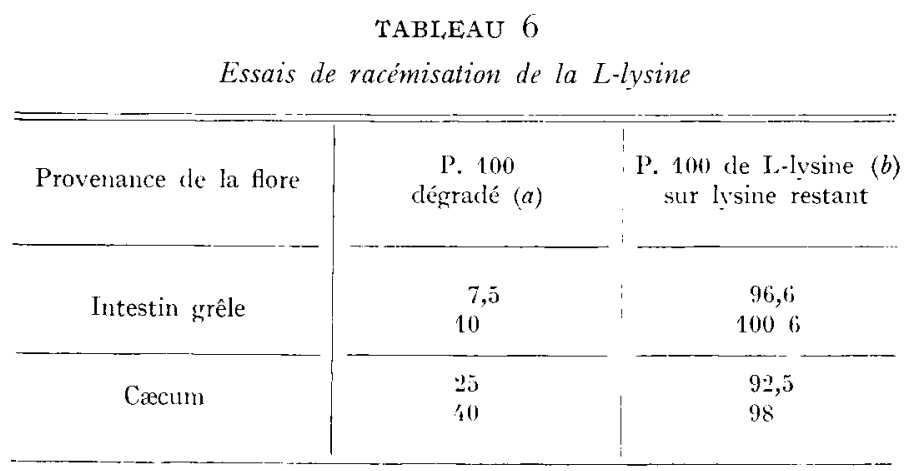

Incubation $16 \mathrm{~h}$ à $37^{\circ} \mathrm{C}$ à $, \mathrm{H} 6,5$.

Substrat $\mathrm{M} / 50$. Flore at $1 / 1$.

a) Mesuré par chromatographie ;

b) Mesuré par la L-lysine décarboxylase.

Chaque essai est effectué sur un lot de 10 animaux.

e) Variations saisonnières et individuelles d'activité catabolique.

Nous avons démontré précédemment (MICHEL, Ig6I a) que la dégradation de l'arginine subissait des variations individuelles et saisonnières importantes. Par 
exemple, le coefficient de dégradation de cette substance ( $\mu 1$ de $\mathrm{NH}_{3}$ formés par heure et par mg de poids sec microbien), passait d'une valeur moyenne de 2 pendant l'hiver à 8 pendant l'été. La formation d'ammoniac à partir de la lysine subit des variations du même ordre, alors que pour la thréonine, le taux de dégradation reste élevé pendant toute l'année (tab1. 7).

TABI,EAU 7

Variations saisonnières du taux de dégradation de la L-lysine et de la L-thréonine

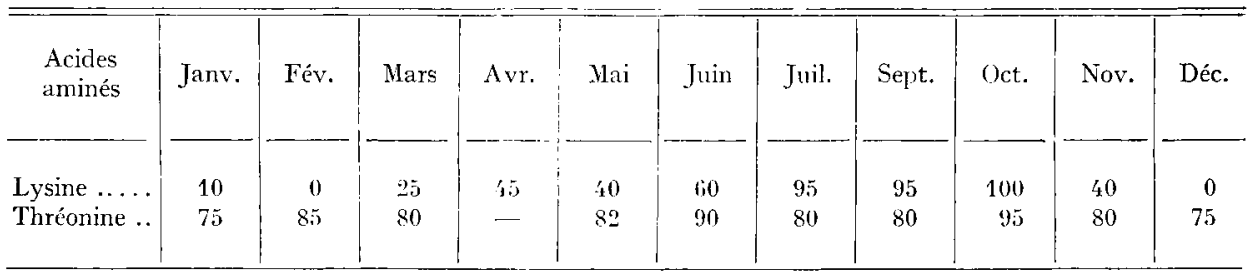

Pourcentage de $\mathrm{N}\left(\mathrm{NH}_{3}\right)$ libéré par rapprort au groupement $\alpha$ aminé. Solution $\mathrm{M} / 50$. Flore au 1/4. $16 \mathrm{~h}$ d'incubation.

Le cas des autres acides aminés essentiels n'a pas été étudié systématiquement, mais on observe également une dispersion importante. En effet, une série de mesures effectuées sur des animaux abattus de décembre à avril a donné les résultats suivants (tabl. 8).

\section{TABLEAU 8}

Désamination des acides aminés essentiels

(forme 1.)

\begin{tabular}{|c|c|c|c|}
\hline \multirow{2}{*}{ Acides aminés } & \multicolumn{2}{|c|}{ P. 100 clégradé } & \multirow{2}{*}{$\begin{array}{c}\text { Écart tylue } \\
\text { en } \%\end{array}$} \\
\hline & Moyenne & Médiane & \\
\hline Thréonine $\ldots \ldots \ldots \ldots \ldots \ldots \ldots$ & $\gamma_{i t}^{\prime}$ & 71 & 15,6 \\
\hline Leucine $\ldots \ldots \ldots \ldots \ldots \ldots \ldots$ & 53,1 & 53,5 & 17,1 \\
\hline Isoleucine $\ldots \ldots \ldots \ldots \ldots \ldots \ldots$ & 53 & 53 & 19,2 \\
\hline Lysine $\ldots \ldots \ldots \ldots \ldots \ldots$ & 35 & 35,5 & 28,4 \\
\hline Valine.$\ldots \ldots \ldots \ldots \ldots \ldots \ldots$ & 30,7 & 36,5 & 11,5 \\
\hline Méthionine.............. & $y_{t}^{\prime}$ & 20,5 & 14,5 \\
\hline Tryptophane ............. & 19 & 20 & 14,5 \\
\hline Proline $\ldots \ldots \ldots \ldots \ldots \ldots \ldots$ & 10 & $7, \bar{i}$ & 9,5 \\
\hline Phénylalanine ............. & 9 & 5 & 10,4 \\
\hline
\end{tabular}

Conditions identiques à celles du tableau précédent.

Ces observations, qui ont été effectuées à une année d'intervalle de celles reportées dans le tableau 3, montrent que l'activité moyenne de la flore, mesurée sur un grand nombre d'animaux, varie peu d'une année à l'autre pour le même acide 
aminé. Il est difficile de déterminer si les variations d'activité sont dues à des changements dans la répartition des diverses espèces microbiennes qui composent la flore ou bien dans leur équipement enzymatique.

\section{f) Catabolisme microbien des amines.}

C'est en été, alors que la flore présente l'activité catabolique maximum à l'égard des acides aminés que la vitesse de dégradation des amines est également la plus élevée. Par exemple, la formation de $\mathrm{NH}_{3}$, à partir de la lysine est faible ou nulle en hiver. Or, nous avons observé récemment que l'acide aminé était quantitativement transformé en cadavérine ( $6 \mathrm{~h}$ d'incubation). En été, cette diamine est dégradée ainsi que d'autres amines (tab1. 9). Si on compare la vitesse de dégradation de ces amines, par rapport à celle de l'acide aminé qui leur donne naissance, on s'aperçoit que cette dernière est presque toujours plus élevée.

\section{TABIEAU 9}

Formation de $\mathrm{NH}_{3}$ à partir de quelques amines

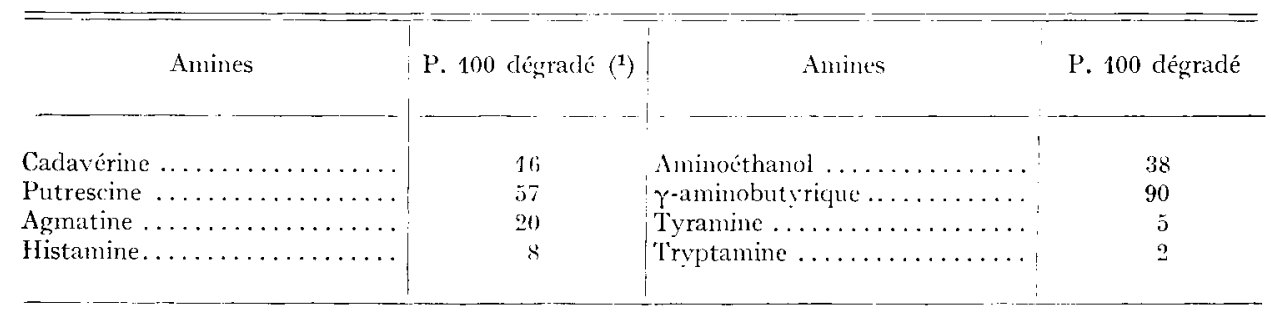

(1) Pourcentage calculé sur un groupement aminé par molécule.

\section{DISCUSSION}

Les résultats de ces essais, qui portent sur trois cents animaux au cours de deux années consécutives montrent l'activité catabolique élevée de la flore intestinale du porc à l'égard des acides aminés de forme $\mathrm{L}$. On observe la formation de diverses amines (cadavérine, putrescine, tyramine, agmatine et tryptamine) à partir des acides aminés correspondants. Ces amines sont les seules qui existent en quantité notable dans les fèces du porc (MICHEI, et al, I964). Lans ce cas, leurs proportions respectives s'accordent assez bien avec ce qui pouvait être prévu d'aprés les essais in vitro, si on considère les vitesses respectives de dégradation de l'acide aminé et de l'amine résultante.

Bien que tous les autres acides aminés ne fournissent pas d'amines, on observe une libération de $\mathrm{CO}_{2}$, mais d'après la cinétique de dégradation, ce serait le résidu carboné, après désamination, qui serait décarboxylé.

Malgré des variations saisonnières et individuelles importantes, l'activité cata- 
bolique moyenne de la flore s'établit aux mêmes valeurs respectives vis-à-vis des différents acides aminés. Par exemple, la vitesse de dégradation de la proline et du tryptophane est toujours inférieure à celle de l'arginine et de la thréonine. Dans l'ensemble, le catabolisme des isomères optiques par la flore concorde assez bien avec l'utilisation de ceux-ci par les organismes supérieurs : travaux d'ALBANESE (I945) sur l'histidine, de Gordon et SIZER (I955) sur la méthionine, de CALET (Ig60) sur la lysine.

Nos résultats sont peu différents de ceux de LEWIS et EMERY (Ig62) qui étudiaient le catabolisme des formes $\mathrm{L}$ et $\mathrm{DL}$ des acides aminés par la flore du rumen de la vache. Pour les dérivés $\mathrm{L}$, le classement des vitesses de dégradation s'établit à peu près dans le même ordre que chez le porc. Seule différence, l'acide D-aspartique n'était pas métabolisé.

En ce qui concerne la lysine, le fait que la flore ne dégrade pas l'isomère I) exclut a priori 1'hypothèse d'une racémisation de cette substance : s'il y avait forma-

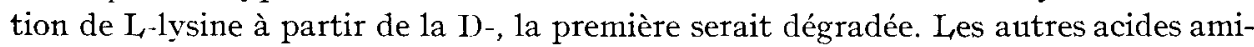
nés essentiels se comportent de la même manière, sauf la méthionine.

L'activité catabolique potentielle de la flore intestinale déterminée in vitro ne s'exerce vraisemblablement qu'en partie au niveau intestinal. Chez l'animal non diarrhéique, le taux de dégradation des substances azotées dans l'estomac, le duodénum et le jéjunum, est limité d'abord par la faible concentration des germes, bien que le taux d'acides aminés libres soit élevé. I)e plus, dans ces segments intestinaux, les germes en phase anabolique dégradent peu les acides aminés. Ce serait à partir de 1'iléon, ainsi que l'ont montré LARSON et HILL, (I960), qu'une activité notable peut être détectée. A partir du cæcum, l'activité catabolique élevée est limitée par la faible teneur en acides aminés libres du contenu. Lorsque le transit intestinal est accéléré, par exemple au cours des diarrhées, l'excrétion fécale des acides aminés et de leurs produits de dégradation devient beaucoup plus importante. Les observations qualitatives effectuées chez le porcelet (Micher, et al., 1965) ont pu être confirmées de manière quantitative chez le veau (travaux en cours).

A partir du cæcum, une partie importante des matières azotées du contenu se trouve sous forme de corps microbiens. L'absorption des acides aminés à ce niveau est peu probable, si on se base sur les résultats obtenus in vitro. En effet, l'autolyse est peu rapide et tout l'azote des corps microbiens autolysés se retrouve sous forme d'ammoniac. Il reste à déterminer si la flore joue un rôle direct ou indirect, sur les desquamations de la muqueuse intestinale et la digestion des substances résultantes. En effet, la présence de la flore intestinale se traduit par des modifications de 1'histologie de la muqueuse, du moins chez les mammifères (rats, souris, cobayes). Comparés aux animaux sans germes, les animaux normaux présentent un épaississement des parois intestinales, une augmentation de la longueur de l'intestin grêle, ainsi qu'un cæcum d'une taille beaucoup plus faible (voir la revue de LuCKEY, I963). La nature des substances responsables de ces modifications n'a pas encore été déterminée. Il en est de même en ce qui concerne la connaissance exacte des substances dépressives de la croissance. Mais diverses preuves indirectes permettent de supposer que cette action dépressive s'exerce par le relais du catabolisme azoté de la flore (voir la discussion de ce problème, Françors et MICHEI, Ig64).

Les processus cataboliques prenant une importance particulière dans les cas 
de diarrhée, il importe de déterminer si les produits de dégradation formés en quantité importante sont la cause de celle-ci ou la manifestation d'un transit accéléré. Les recherches en cours visent à répondre à ces questions.

\author{
Reçu pour publication en octobre 1965.
}

\title{
SUMMARY
}

\author{
METABOLISM OF THE INTESTINAL FLORA OF THE PIG. \\ BREAKDOWN OF L- AND D-FORMS OF AMINO ACIDS
}

I. Total microbial flora taken from the caecum of pigs at slaughter was incubated in vitro with each of the amino acids to study catabolism of the acids.

2. Conditions for incubation were chosen to resemble as cosely as possible the conditions in the intestinal contents, with $\mathrm{plI}$ about 6 , th about $-250 \mathrm{mV}$ and microbial concentration $2 \mathrm{~g}$ dry weight per litre. All the $\mathrm{L}$ forms of the amino acids were broken down by deamination and decarboxylation. The rate of breakdown depended on the particular amino acid; serine $100 \mathrm{p}$. Ioo in I6 hours, glutamic acid 100 , histidine 98 , aspartic acid 78 , arginine 72 , threonine $62 \mathrm{p}$. 100 . The other amino acids were broken down more slowly, from 20 to $50 \mathrm{p}$. 100 .

3. Substances resulting from the catabolism were $\mathrm{NH}_{3}, \mathrm{CO}_{2}$, eventually $\mathrm{H}_{2} \mathrm{~S}$, different organic acids and amines. Lysine $\rightarrow$ cadaverine ; ornithine $\rightarrow$ putrescine ; arginine $\rightarrow$ citrulline, agmatine, putrescine and ornithine; histidine $\rightarrow$ histamine and urocanic acid ; tyrosine $\rightarrow$ tyramine ; tryptophan $\rightarrow$ indole and tryptamine. The other amino acids were first deaminated then the carbon residues were broken down in their turn.

4. Only the $\mathrm{L}$ forms of the essential amino acids were broken down by the flora, to the exclusion of the D forms, except in the case of D-methionine. D forms of the semi-essential and other unclassified amino acids were broken down, but more slowly than the $\mathrm{L}$, forms.

5. The catabolic activity, estimated in a large number of animals, differed widely between seasons; the rate of breakdown of arginine was four times as great in summer as in winter.

6. All the products of microbial catabolism of amino acids were found in the intestine of the live animal. 'Their eventual effects on the metabolism of the host are discussed.

\section{RÉFÉRENCES BIBLIOGRAPHIQUES}

Albanese A. A., Frankston J. E., Irby V., ig45. The utilization of $d$-aminoacids by man. J. Biol. Chem., 160, $44 \mathrm{I}-447$.

Calet C., ig6o. Contribution à l'étude de l'efficacité des formes $\mathrm{L}$ et $\mathrm{DL}$ de la lysine pour la croissancé du poussin. Ann. Nutr. Aliment., 14, 287-29o.

Conway E. J., 1957. Microdiffusion analysis and volumetric error. Crosby Lockwood, London, 4 th ed.

Dickinson A., 1961. Travaux de la Commission des antibiotiques du C. N. E. R. N. A. Ann. Nitr. Alim. 15, $84-90$.

Ex-Sinzly K., 1952. Degradation of protein in the rumen of the sheep. Biochem. J., 51, 640-653.

Françors A. C., Mrchel M. C., I960. Effets métaboliques de certaines flores digestives. Conséquences nutritionnelles. Cahiers du Collège de Médecine, 12, 949-955.

François A. C., Miciele M. C., I964. Flore du tractus digestif et nutrition de l'hôte. Rôle des antibiotiques. Symposium sur la digestion des protéines. Glasgrow.

GALE E. F., 1946. The bacterial aminoacid decarboxylase. Advances in Ensymology, 6, 1-32.

Gordon R. S., SIzER I. W., I955. The biological equivalence of methionine hydroxy analogue. Poultry Sci., 34, I 198.

Huang I1. 'T., Davisson J. W., 1958. Distribution of lysine racemase in bacteria. J. Bacleriol., 76, 495 .

KNIVEetT J.A., I960. The bacterial degradation of arginine. Biochimie comparée des acides aminés basiques, Concarneau. 'Éditions du C. N. R. S., $2+3-260$.

Lakson N. L., HiLl E. G., I960. Amine formation and metabolic activity of microorganisms in the ileum of young swine fed chlortetracycline. J. Bacteriol., 80, I88-192. 
LewIs. D., 1955. Amino-acid metalsolism in the rumen of the sheep. Brit. J. Nutr., 9, 21 (5)-23I.

Lewis T. R., FMerY R. S., 1962. Relative desamination rates of amino-acids by rumen microorganisms. J. Dairy Sci., 45, 765-768.

LuCKEY T. D., I963. Germ-free life and gnotobiology: Acalemic Press., New York.

Ludvigsen J. B., Thorber G., rght. Fermentation processes in the ciecum of pigs. VIII Inlernalionaler Tiersuchlkongress, IIambourg.

Metankowicz J., Jonanssox K. R., 1955. Formation of amines by intestinal microorganisms and the influence of tetracycline. J. Exper. Med.., 101, 507-517.

Michri M. C., тg6ra. Activité métabolique de la flore totale isolée de l'intestin du Porc. Rôle des différentes especes microbiennes. Ann. Biol. anim. Bioch. Biophy's, 1, г6-28.

Michel M. C., rg6r c. Dosage de l'azote aminé dans quelques liquides biologiques. Ann. Biol. anim. Bioch. Biophys., 1, 248-255.

Micher. M. C., SaCQUet E., 1965 . Acides aninés du contenu intestimal et leurs produits de décomposition chez le rat aseptique et conv'ntionnel. Inlernationales Mikroökologisches. Symposium. l'ostdiun (sous presse).

Michel M. C., Jorandet C., Salmox-Legiagnetr E., Almaitre A., Frasçots A. C., 1964. Influence de l'acrylate de sodium sur la croissance du porcelet. Ann. Zootech., 13, 34I-350.

Moore J. II., TYLer C., 1955. Studies on the intestinal absorption and excretion of calcium and phosphorus in the pig. Brit. J. Nutr., 9, 8г.

Wurmser R., 1935. I'électroactivité dans la chimie des cellules. Hermann et Cie, Paris. 